\title{
WEIGHTED GREATEST COMMON DIVISORS AND WEIGHTED HEIGHTS
}

\author{
L. BESHAJ, J. GUTIERREZ, T. SHASKA
}

\begin{abstract}
We introduce the weighted greatest common divisor of a tuple of integers and explore some of its basic properties. Furthermore, for a set of heights $\mathfrak{w}=\left(q_{0}, \ldots, q_{n}\right)$, we use the concept of the weighted greatest common divisor to define a height $\mathfrak{h}(\mathfrak{p})$ on weighted projective spaces $\mathbb{W P}_{\mathfrak{w}}^{n}(k)$. We prove some of the basic properties of this weighted height, including an analogue of the Northcott's theorem for heights on projective spaces.
\end{abstract}

\section{INTRODUCTION}

Most of the computations with genus 2 curves or genus 3 hyperelliptic curves, whether occurring in number theory, mathematical physics, cryptography, or any other area, involve the corresponding tuple of invariants of binary forms. An isomorphism class of such curves correspond to a projective point $\left[J_{q_{0}}: \cdots: J_{q_{n}}\right]$ of modular invariants with degrees $q_{0}, \ldots, q_{n}$ respectively. Of course this is true for all hyperelliptic or even superelliptic curves of any genus. In most of these computations picking the point $\left[J_{q_{0}}: \cdots: J_{q_{n}}\right]$ with smallest coordinates is desirable; see for example computations in [15, 12, [7] or all the algorithms in cryptography for genus $g=2$ and $g=3$ hyperelliptic curves. So how can we pick the point with smallest coordinates or have some ordering on these moduli points in some reasonable way? Since the ring of invariants of binary forms is a graded ring, the answer is equivalent to introducing some concept of the greatest common divisor for weighted projective spaces similar to that of the gcd of a tuple of integers in the projective space. If possible we would like to extend the analogy and introduce some concept of height in a weighted projective space similarly to the height in a regular projective space, which would make the ordering of points in a weighted projective space possible. The goal of this paper is to suggest a way to handle both of these questions.

In [16] was introduced the idea of the weighted common divisor on a tuple of integers with different weights, which was called the weighted greatest common divisor and denoted by wgcd. In section 2 we give a precise definition of the concept of the weighted greatest common divisor and some of its properties. While the idea of the weighted greatest common divisor seems natural, surprisingly it has not appeared before in the literature. Questions still remain on efficient ways of computing such common divisor or whether such weighted greatest common divisor has similar properties as the regular greatest common divisor in more general rings.

For a a set of weights $\mathfrak{w}=\left(q_{0}, \ldots, q_{n}\right)$ and a number field $K$, the weighted greatest common divisor $\operatorname{wgcd}(\mathbf{x})$ of a tuple $\mathbf{x}=\left(x_{0}, \ldots, x_{n}\right) \in \mathcal{O}_{K}^{n+1}$ is defined as the largest integer $d \in \mathcal{O}_{K}$ such that $d^{q_{i}}$ divides $x_{i}$, for all $i=1, \ldots, n$. The absolute greatest common divisor $\overline{\mathrm{wgcd}}(\mathbf{x})$ is defined as the largest real number $d$ 
such that $d^{q_{i}}$ divides $x_{i}$, for all $i=1, \ldots, n$. Lem. 3 shows that this definitions are precise.

In section 3 we apply this method to normalize points in weighted projective spaces. A point $\mathfrak{p}=\left[x_{0}, \ldots, x_{n}\right.$ in the weighted projective space $\mathbb{W P}_{\mathfrak{w}}^{n}(K)$ is said to be normalized if $\operatorname{wgcd}\left(x_{0}, \ldots, x_{n}\right)=1$ and absolutely normalized if $\overline{\operatorname{wgcd}}\left(x_{0}, \ldots, x_{n}\right)=1$. It turns out that these normalizations are unique up to multiplication by a root of unity (cf. Lem. 7). Moreover, such normalization is unique for well-formed weighted projective spaces. Normalizing point in a weighted projective space this way gives a very efficient way of storing points in such spaces. This idea was used in [3] and 2] to study the moduli space of genus 2 and genus 3 hyperelliptic curves.

In section 4 we shift our attention to introducing heights in weighted projective spaces. The concept of height on a variety $\mathcal{A}$ over a number field $K$ is a function $H$ : $\mathcal{A}(K) \rightarrow \mathbb{R}$ whose value at a point $P \in \mathcal{A}(K)$ measures the arithmetic complexity of $P$. There are two properties that one would want in a height function: i) there are only finitely many points of bounded height, ii) geometric properties are somewhat preserved.

Heights on projective spaces are well known in the literature; see [20, [19, [5] among many others. For a point $P \in \mathbb{P}^{n}(\mathbb{Q})$, we take integer projective coordinates $P=\left[x_{0}: \cdots: x_{n}\right]$ with $\operatorname{gcd}\left(x_{0}, \ldots, x_{n}\right)=1$, then the height is defined as

$$
H(P)=\max \left\{\left|x_{0}\right|, \ldots,\left|x_{n}\right|\right\} .
$$

The definition can be extended to any number field $K$ as follows

$$
H_{K}(P)=\prod_{v \in M_{K}} \max \left\{\left|x_{0}\right|_{v}^{n_{v}}, \ldots,\left|x_{n}\right|_{v}^{n_{v}}\right\} .
$$

where $M_{K}$ is the set of norms in $K$ and $n_{v}$ the local degree $\left[K_{v}: \mathbb{Q}_{v}\right]$. As an immediate consequence of the definition is the Northcott's theorem, which says that there are only finitely many points $P \in \mathbb{P}^{n}(K)$, with height bounded by a constant $B$. A corollary of this statement is the Kronecker's theorem which says that for any $\alpha \in K^{\star}, H_{K}(\alpha)=1$ if and only if $\alpha$ is a root of unity. In other words, there are only finitely many points of bounded height and bounded degree.

Let $V_{K}$ be a projective subvariety of $\mathbb{P}^{n}(K)$ and $S \subset V_{K}$. In arithmetic, height functions are used in two main ways: i) To show that $S$ is finite, it is enough to show that it is a set of bounded height, ii) if $S$ is infinite, determine its density by estimating the growth of the counting function $N(S, B)=\#\left\{P \in S: H_{K}(P) \leq\right.$ $B\}$. The size of the set of points in $\mathbb{P}^{n}(K)$ is estimated by Schanuel's theorem.

Weil extended the definition of height to all projective varieties via ample divisors and provided an important connection between geometry and arithmetic. Néron and Tate introduced canonical heights for Abelian varieties. Perhaps one of the most popular uses of the machinery of heights is the proof of the MordellWeil theorem: For any Abelian variety $\mathcal{A}_{K}$, the set of $K$-points $\mathcal{A}(K)$ is a finitely generated Abelian group. The main goal of this paper is to investigate how the machinery of heights for projective spaces can be extended to weighted projective spaces. Whether the weighted projective height introduced here can be interpreted in terms of blowups, along the lines of [20, will be the focus of future investigation.

Let $\mathfrak{w}=\left(q_{0}, \ldots, q_{n}\right)$ be a set of heights and $\mathbb{W P}^{n}(K)$ the weighted projective space over a number field $K$ and $M_{K}$ the set of places of $K$. Let $\mathfrak{p} \in \mathbb{W}^{n}(K)$ a 
point such that $\mathfrak{p}=\left[x_{0}, \ldots, x_{n}\right]$. We define the weighted height of $\mathfrak{p}$ as

$$
\mathfrak{h}_{K}(\mathfrak{p}):=\prod_{v \in M_{K}} \max \left\{\left|x_{0}\right|_{v}^{\frac{n_{v}}{q_{0}}}, \ldots,\left|x_{n}\right|_{v}^{\frac{n_{v}}{q_{n}}}\right\}
$$

The weighted logarithmic height of the point $\mathfrak{p}$ is defined as follows

$$
\mathfrak{h}_{K}^{\prime}(\mathfrak{p}):=\log \mathfrak{h}_{K}(\mathfrak{p})=\sum_{v \in M_{K}} \max _{0 \leq j \leq n}\left\{\frac{n_{v}}{q_{j}} \cdot \log \left|x_{j}\right|_{v}\right\} .
$$

We prove that $\mathfrak{h}_{K}(\mathfrak{p})$ is well defined and $\mathfrak{h}_{K}(\mathfrak{p}) \geq 1$. Moreover, if $K=\mathbb{Q}(\overline{\text { wgcd }}(\mathfrak{p}))$ then similarly to the projective space,

$$
\mathfrak{h}_{K}(\mathfrak{p})=\max _{0 \leq j \leq n}\left\{\left|x_{j}\right|_{\infty}^{1 / q_{j}}\right\}
$$

when $\mathfrak{p}$ is normalized and if $L / K$ is a finite extension, then $\mathfrak{h}_{L}(\mathfrak{p})=\mathfrak{h}_{K}(\mathfrak{p})^{[L: K]}$. The absolute height of a point $\mathfrak{p} \in \mathbb{W}^{n}(K)$ is defined as $\tilde{\mathfrak{h}}: \mathbb{W} \mathbb{P}^{n}(\overline{\mathbb{Q}}) \rightarrow[1, \infty)$ for $\tilde{\mathfrak{h}}(\mathfrak{p})=\mathfrak{h}_{K}(\mathfrak{p})^{1 /[K: \mathbb{Q}]}$. It turns out that for weighted heights $\mathbb{Q}(\overline{\text { wgcd }}(\mathfrak{p}))$ plays the role that the base field $\mathbb{Q}$ plays for regular projective height, see Prop. 5 , This is no surprise since the greatest common divisor is in $\mathbb{Q}$ for projective heights. We are also able to consider the weighted heights through the Weil height, via the map $\phi: \mathbb{W}_{\mathfrak{w}}^{n}(K) \rightarrow \mathbb{P}^{n}(K)$, where

$$
\left[x_{0}, \ldots, x_{n}\right] \rightarrow\left[x_{0}^{\frac{q}{q_{0}}}, \ldots, x_{n}^{\frac{q}{q_{n}}}\right]
$$

where $q=q_{0} \cdots q_{n}$. Then $\mathfrak{h}(\mathfrak{p})=H\left(\phi(\mathfrak{p})^{\frac{1}{q}}\right.$, see Lem. 9 As in the projective space the weighted height is invariant under Galois conjugation. In other words, for $\mathfrak{p} \in \mathbb{W P}^{n}(\overline{\mathbb{Q}})$ and $\sigma \in G_{\mathbb{Q}}$ we have $\mathfrak{h}\left(\mathfrak{p}^{\sigma}\right)=\mathfrak{h}(\mathfrak{p})$ (cf. Lem. 10). In Thm. 10 we prove an analogue of Northcott's theorem for weighted heights.

The weighted height seem to provide a powerful tool in studying the arithmetic properties of the weighted projective spaces. This could lead to many interesting results in many applications of such spaces.

Aknowledgments: We want to thank J. Silverman and J. Ellenberg for insightful comments and suggestions which significantly improved this paper.

\section{Weighted GREATEST COMMON DIVISORS}

Let $\mathbf{x}=\left(x_{0}, \ldots x_{n}\right) \in \mathbb{Z}^{n+1}$ be a tuple of integers, not all equal to zero. Their greatest common divisor, denoted by $\operatorname{gcd}\left(x_{0}, \ldots, x_{n}\right)$, is defined as the largest integer $d$ such that $d \mid x_{i}$, for all $i=0, \ldots, n$.

The concept of the weighted greatest common divisor of a tuple for the ring of integers $\mathbb{Z}$ was defined in [16]. Let $q_{0}, \ldots, q_{n}$ be positive integers. A set of weights is called the ordered tuple $\mathfrak{w}=\left(q_{0}, \ldots, q_{n}\right)$.

Denote by $r=\operatorname{gcd}\left(q_{0}, \ldots, q_{n}\right)$ the greatest common divisor of $q_{0}, \ldots, q_{n}$. A weighted integer tuple is a tuple $\mathbf{x}=\left(x_{0}, \ldots, x_{n}\right) \in \mathbb{Z}^{n+1}$ such that to each coordinate $x_{i}$ is assigned the weight $q_{i}$. We multiply weighted tuples by scalars $\lambda \in \mathbb{Q}$ via

$$
\lambda \star\left(x_{0}, \ldots, x_{n}\right)=\left(\lambda^{q_{0}} x_{0}, \ldots, \lambda^{q_{n}} x_{n}\right)
$$


For an ordered tuple of integers $\mathbf{x}=\left(x_{0}, \ldots, x_{n}\right) \in \mathbb{Z}^{n+1}$, whose coordinates are not all zero, the weighted greatest common divisor with respect to the set of weights $\mathfrak{w}$ is the largest integer $d$ such that

$$
d^{q_{i}} \mid x_{i}, \text { for all } i=0, \ldots, n \text {. }
$$

The first natural question arising from this definition is to know if such integer $d$ does exist for any tuple $\mathbf{x}=\left(x_{0}, \ldots, x_{n}\right) \in \mathbb{Z}^{n+1}$. Clearly, it does exist because $x_{i} \leq d^{q_{i}}$ for all $i=0, \ldots, n$ and the largest integer is unique. We will denote by $\operatorname{wgcd}\left(x_{0}, \ldots, x_{n}\right)=\operatorname{wgcd}(\mathbf{x})$.

Given integer $a$ and non-zero integer $b$, the integer part of the real number $\frac{a}{b}$ is denote by $\left\lfloor\frac{a}{b}\right\rfloor$, that is, it is the unique integer satisfying:

$$
a=\left\lfloor\frac{a}{b}\right\rfloor b+r, \quad 0 \leq r<b .
$$

The next result provides an algorithm to compute the weighted greatest common divisor.

Proposition 1. For a weighted integer tuple $\mathbf{x}=\left(x_{0}, \ldots, x_{n}\right)$ with weights $\mathfrak{w}=$ $\left(q_{0}, \ldots, q_{n}\right)$ let the factorization of the integers $x_{i},(i=0, \ldots, n)$ into primes:

$$
x_{i}=\prod_{j=1}^{t} p_{j}^{\alpha_{j, i}}, \quad \alpha_{j, i} \geq 0, j=1, \ldots, t
$$

Then, the weighted greatest common divisor $d=\operatorname{wgcd}(\mathbf{x})$ is given by

$$
d=\prod_{j=1}^{t} p_{j}^{\alpha_{j}}
$$

where,

$$
\alpha_{j}=\min \left\{\left\lfloor\frac{\alpha_{j, i}}{q_{i}}\right\rfloor, i=0, \ldots, n\right\} \text { and } j=1, \ldots, t
$$

Proof. If $d^{q_{i}} \mid x_{i}$, then $d$ should be of the form $\prod_{j=1}^{t} p_{j}^{\beta_{j}}$ for certain integers $\beta_{j} \geq 0$. On the other hand, for every prime $p_{j}$ and since $d^{q_{i}} \mid x_{i}$, then

$$
\beta_{j} \leq \frac{\alpha_{j, i}}{q_{i}}, i=0, \ldots, n .
$$

Now, the proof is straightforward.

In the next we illustrate the method by a toy example:

Example 1. Consider the set of weights $\mathfrak{w}=(3,2)$ and the tuple

$$
\mathbf{x}=(1440,700)=\left(2^{5} \cdot 3^{2} \cdot 5 \cdot 7^{0}, 2^{2} \cdot 3^{0} \cdot 5^{2} \cdot 7\right) \in \mathbb{Z}^{2} .
$$

Then, $\operatorname{wgcd}(\mathbf{x})=d=2^{\alpha_{1}} \cdot 3^{\alpha_{2}} \cdot 5^{\alpha_{3}} \cdot 7^{\alpha_{4}}$, where

$$
\begin{aligned}
& \alpha_{1}=\min \left\{\left\lfloor\frac{5}{3}\right\rfloor,\left\lfloor\frac{2}{2}\right\rfloor\right\}=1, \quad \alpha_{2}=\min \left\{\left\lfloor\frac{2}{3}\right\rfloor,\left\lfloor\frac{0}{2}\right\rfloor\right\}=0, \\
& \alpha_{3}=\min \left\{\left\lfloor\frac{1}{3}\right\rfloor,\left\lfloor\frac{0}{2}\right\rfloor\right\}=0, \quad \alpha_{4}=\min \left\{\left\lfloor\frac{0}{3}\right\rfloor,\left\lfloor\frac{1}{2}\right\rfloor\right\}=0 .
\end{aligned}
$$

Then $d=2$. 
An integer tuple $\mathbf{x}=\left(x_{0}, \ldots, x_{n}\right) \in \mathbb{Z}^{n+1}$ with $\operatorname{wgcd}(\mathbf{x})=1$ is called normalized. For an integer tuple $\mathbf{x}=\left(x_{0}, \ldots, x_{n}\right)$ exist integers $\left(y_{0}, \ldots, y_{n}\right) \in \mathbb{Z}^{n+1}$ such that

$$
\operatorname{gcd}\left(x_{0}, \ldots x_{n}\right)=x_{0} y_{0}+\cdots+x_{n} y_{n} .
$$

For weights $\mathfrak{w}=\left(q_{0}, \ldots, q_{n}\right)$, we have that $\operatorname{wgcd}(\mathbf{x}) \mid \operatorname{gcd}(\mathbf{x})$, say

$$
\operatorname{gcd}(x)=\lambda \cdot \operatorname{wgcd}(\mathbf{x}) \text {. }
$$

Then,

$$
\operatorname{wgcd}(\mathbf{x})=\left(\frac{x_{0}}{\lambda}\right) y_{0}+\left(\frac{x_{1}}{\lambda}\right) y_{1}+\cdots+\left(\frac{x_{n}}{\lambda}\right) y_{n}=\sum_{i=0}^{n}\left(\frac{x_{i}}{\lambda}\right) y_{i}
$$

Notice that each $\frac{x_{i}}{\lambda}$ is an integer from the definition of the $\operatorname{wgcd}(\mathbf{x})$.

The absolute weighted greatest common divisor of an integer tuple $\mathbf{x}=$ $\left(x_{0}, \ldots, x_{n}\right)$ with respect to the set of weights $\mathfrak{w}=\left(q_{0}, \ldots, q_{n}\right)$ is the largest real number $d$ such that

$$
d^{q_{i}} \in \mathbb{Z} \quad \text { and } \quad d^{q_{i}} \mid x_{i}, \text { for all } i=0, \ldots n .
$$

Again, the natural question arising from this new definition is to know if such real number $d$ does exist for any tuple $\mathbf{x}=\left(x_{0}, \ldots, x_{n}\right) \in \mathbb{Z}^{n+1}$. Since $x_{i} \leq d^{q_{i}}$ and there are a finite number of divisors of $x_{i}$, for all $i=0, \ldots, n$, so we are looking for the largest real number of finite set of numbers and, the largest is unique. We will denote by the absolute weighted greatest common divisor by $\overline{\mathrm{wgcd}}\left(x_{0}, \ldots, x_{n}\right)$.

In order to provide a method to compute the $\overline{\operatorname{wgcd}}\left(x_{0}, \ldots, x_{n}\right)$, we need the following technical elementary result.

Lemma 1. Let $d \in \mathbb{R}^{+}$a positive real number. If there exists a positive integer $m$ such that $d^{m}$ is a positive integer, then $d=z^{1 / m}$ for some positive integer $z$. Moreover if $m$ is the smallest integer such that $d^{m}$ is a positive integer, then any positive integer $q$ verifying $d^{q}$ is a positive integer, is a multiple of $m$.

The next result provides a method to compute the absolute weighted greatest common divisor:

Proposition 2. For a weighted integer tuple $\mathbf{x}=\left(x_{0}, \ldots, x_{n}\right)$ with weights $\mathfrak{w}=$ $\left(q_{0}, \ldots, q_{n}\right)$ let the factorization of the integers $x_{i},(i=0, \ldots, n)$ into primes:

$$
x_{i}=\prod_{j=1}^{t} p_{j}^{\alpha_{j, i}}, \quad \alpha_{j, i} \geq 0, j=1, \ldots, t
$$

Then, the absolute weighted greatest common divisor $d=\overline{\mathrm{wgcd}}(\mathbf{x})$ is given by

$$
d=\left(\prod_{j=1}^{t} p_{j}^{\alpha_{j}}\right)^{\frac{1}{q}}
$$

where, $q=\operatorname{gcd}\left(q_{0}, \ldots, q_{n}\right), \quad q_{i}=q \cdot \bar{q}_{i}$ and

$$
\alpha_{j}=\min \left\{\left\lfloor\frac{\alpha_{j, i}}{\bar{q}_{i}}\right\rfloor, i=0, \ldots, n\right\} \text { and } j=1, \ldots, t .
$$


Proof. From Lem. 1 we have that $d^{q} \mid x_{i}, i=0, \ldots, n$. Then $d$ should be of the form $d=\left(\prod_{j=1}^{t} p_{j}^{\beta_{j}}\right)^{\frac{1}{q}}$ for certain integers $\beta_{j} \geq 0$. On the other hand, for every prime $p_{j}$ and since $d^{q_{i}} \mid x_{i}$, then

$$
\beta_{j} \leq \frac{\alpha_{j, i}}{\bar{q}_{i}}, i=0, \ldots, n .
$$

Again, the rest of the proof is immediate.

Example 2. Consider the set of weights $\mathfrak{w}=(6,8)$ and the tuple

$$
\mathbf{x}=\left(2^{15} \cdot 5^{12}, 2^{26} \cdot 5^{13}\right) \in \mathbb{Z}^{2} .
$$

Then $q=\operatorname{gcd}(6,8)=2, p_{1}=2, p_{2}=5, t=2$ and $\bar{q}_{1}=3, \bar{q}_{2}=4$. Then, $\overline{\operatorname{wgcd}}(\mathbf{x})=$ $d=\left(2^{\alpha_{1}} \cdot 5^{\alpha_{2}}\right)^{\frac{1}{2}}$, where

$$
\alpha_{1}=\min \left\{\left\lfloor\frac{15}{3}\right\rfloor,\left\lfloor\frac{26}{4}\right\rfloor\right\}=5, \quad \alpha_{2}=\min \left\{\left\lfloor\frac{12}{3}\right\rfloor,\left\lfloor\frac{13}{4}\right\rfloor\right\}=3 .
$$

Hence $d=2^{\frac{5}{2}} \cdot 5^{\frac{3}{2}}=\sqrt{2^{5} \cdot 5^{3}}$. On the other hand, $\operatorname{wgcd}(\mathbf{x})=2^{2} \cdot 5$. As expected, $\operatorname{wgcd}(\mathbf{x}) \leq \overline{\operatorname{wgcd}}(\mathbf{x})$.

The next example comes from the theory of invariants of binary sextics.

Example 3. Consider the set of weights $\mathfrak{w}=(2,4,6,10)$ and a tuple

$$
\mathbf{x}=\left(3 \cdot 5^{2}, 3^{2} \cdot 5^{4}, 3^{3} \cdot 5^{6}, 3^{5} \cdot 5^{10}\right) \in \mathbb{Z}^{4} .
$$

Then, $\operatorname{wgcd}(\mathbf{x})=5$ and $\overline{\operatorname{wgcd}}(\mathbf{x})=5 \cdot \sqrt{3}$.

An integer tuple $\mathbf{x}$ with $\overline{\operatorname{wgcd}}(\mathbf{x})=1$ is called absolutely normalized. We summarize in the following lemma.

Lemma 2. For any weighted integral tuple $\mathbf{x}=\left(x_{0}, \ldots, x_{n}\right) \in \mathbb{Z}^{n+1}$ such that $\mathfrak{w}\left(x_{i}\right)=q_{i}, i=0, \ldots, n$, the tuple $\mathbf{y}=\frac{1}{\operatorname{wgcd}(\mathbf{x})} \star \mathbf{x}$, is integral and normalized. Moreover, the tuple $\overline{\mathbf{y}}=\frac{1}{\operatorname{wgcd}(\mathbf{x})} \star \mathbf{x}$, is also integral and absolutely normalized.

Normalized tuples are unique up to a multiplication of $q$-root of unity (cf. Lem. 7), where $q=\operatorname{gcd}\left(q_{0}, \ldots, q_{n}\right)$. It is worth noting that a normalized tuple is a tuple with "smallest" integer coordinates (up to multiplication by a unit). We will explore this idea of the "smallest coordinates" in the coming sections.

There are a few natural questions that arise with the weighted greatest common divisor of a tuple of integers. We briefly discuss the two main ones:

Problem 1: The greatest common divisor can be computed in polynomial time using the Euclidean algorithm. Determine the fastest way to compute the weighted greatest common divisor and the absolute weighted greatest common divisor.

Problem 2: The greatest common divisor is uniquely determined for unique factorization domains. Define the concept of the weighted greatest common divisor in terms of ring theory and determine the largest class of rings where it is uniquely defined (up to multiplication by a unit). 
2.1. Complexity of computing the weighted greatest common divisor. Let $\mathbf{x}=\left(x_{0}, \ldots, \ldots, x_{n}\right) \in \mathbb{Z}^{n+1}$ and weights $\mathfrak{w}=\left(q_{0}, \ldots, q_{n}\right)$. Then Prop. 1 and Prop. 2 provide a method to compute $\operatorname{wgcd}(\mathbf{x})$ and $\overline{\mathrm{wgcd}}(\mathbf{x})$ (respectively) for weights $\mathfrak{w}=\left(q_{0}, \ldots, q_{n}\right)$. In both, we have to compute the integer factorization into primes of all elements of the tuple $\mathbf{x}$. Of course, this is not very efficient comparing with the computation of $\operatorname{gcd}(\mathbf{x}))$. On the other hand, there are several indications that we can not avoid factoring. For instance, we have that $\operatorname{wgcd}\left(0, \ldots, 0, x_{n}\right)$ is wgcd $\left(x_{n}\right)$, then we are looking for the largest factor $d$ of $x_{n}$ such that $d^{q_{n}}$ divides $x_{n}$.

Alternatively, we can factor only an integer, instead of $n+1$, and then recombining factors in an appropriate and clever way gives us the following.

Lemma 3. With the above notation, let $g=\operatorname{gcd}\left(x_{0}, \ldots, x_{n}\right)$ and $g=\prod_{i=1}^{r} p_{i}^{s_{i}}$ its prime factorization.

(1) For $i=1, \ldots, r$, let

$$
\beta_{i}=\min \left\{\left\lfloor\frac{s_{i}}{q_{j}}\right\rfloor: j=0, \ldots, n\right\} .
$$

Then, the weighted greatest common divisor $d=\operatorname{wgcd}(\mathbf{x})$ is given by

$$
d=\prod_{i=1}^{r} p_{i}^{\alpha_{i}}
$$

where $\alpha_{i}$ are the largest integers such that $d^{q_{i}}$ divides $x_{i}$ and $\alpha_{i} \leq \beta_{i}$.

(2) Let $q=\operatorname{gcd}\left(q_{0}, \ldots, q_{n}\right), q_{j}=q \cdot \bar{q}_{j}, j=0, \ldots, n$ and for $i=1, \ldots, r$ let

$$
\beta_{i}=\min \left\{\left\lfloor\frac{s_{i}}{\bar{q}_{j}}\right\rfloor, j=0, \ldots, n\right\}
$$

Then, the absolute weighted greatest common divisor $d=\overline{\mathrm{wgcd}}(\mathbf{x})$ is

$$
d=\left(\prod_{i=1}^{r} p_{i}^{\alpha_{i}}\right)^{\frac{1}{q}}
$$

where $\alpha_{i}$ are the largest integers such that $d^{q_{i}}$ divides $x_{i}$ and $\alpha_{i} \leq \beta_{i}$.

Proof. To prove 1) we have that $d^{q_{i}}$ divides $x_{i}$, then $d$ divides $x_{i}$ and it implies $d$ divides $g$. Now, the proof is straightforward. To prove 2) we have that $d^{q_{i}}$ divides $x_{i}$ and from Lem. 1, $d^{q}$ divides $x_{i}$ and it implies that $d^{q}$ divides $g$. The rest proof is immediate.

It is well known that the number of divisors $D(m)$ of integer $m$ is $m^{o(1)}$. So, in the worst case the previous result Lem. 3 get an exponential time complexity.

2.2. Weighted greatest common divisor over general rings. Let $R$ be a commutative ring with identity. Consider the set of weights $\mathfrak{w}=\left(q_{0}, \ldots, q_{n}\right)$ as in the previous section and a tuple $\mathbf{x} \in R^{n+1}$. For any $\alpha \in R$, the ideal generated by $\alpha$ is denoted by $(\alpha)$. The weighted greatest common divisor ideal is defined as

$$
\mathfrak{J}(\mathbf{x})=\bigcap_{\left(\mathfrak{p}^{q_{i}}\right) \supset\left(x_{i}\right)} \mathfrak{p}
$$

over all primes $\mathfrak{p}$ in $R$. If $R$ is a PID then the $\operatorname{wgcd}(\mathbf{x})$ is the generator of the principal ideal $\mathfrak{J}(\mathbf{x})$. In general, for $R$ a unique factorization domain, for any point 
$x=\left(x_{1}, \ldots, x_{n}\right) \in R^{n}$ we let $r=\operatorname{gcd}\left(x_{0}, \ldots, x_{n}\right)$. Factor $r$ as a product of primes, say $r=u \cdot \prod_{i=1}^{s} \mathfrak{p}_{i}$, where $u$ is a unit and $\mathfrak{p}_{1}, \ldots \mathfrak{p}_{s}$ are primes. Then the weighted $\operatorname{gcd} \operatorname{wgcd}(\mathbf{x})$ is defined as

$$
\operatorname{wgcd}(\mathbf{x})=\prod_{\substack{i=1 \\ p^{q_{i}} \mid x_{i}}}^{s} \mathfrak{p}
$$

Thus, the weighted gcd (as the common gcd) is defined up to multiplication by a unit. The absolute weighted greatest common divisor ideal is defined as

$$
\overline{\mathfrak{J}}(\mathbf{x})=\bigcap_{\left(\mathfrak{p}^{\frac{q_{i}}{r}}\right) \supset\left(x_{i}\right)} \mathfrak{p}
$$

over all primes $\mathfrak{p}$ in $R$.

The above definitions can be generalized to GCD domains. An integral domain $R$ is called a GCD domain if any two elements of $R$ have a greatest common divisor. Examples of GCD-domains include unique factorization domains and valuation domains, see 13 for more details.

2.3. Generalized weighted greatest common divisors. Following on the ideas of 20 we give a brief review of the generalized greatest common divisors and how they can be defined for weighted greatest common divisors as well. Let $k$ be a number field, $\mathcal{O}_{k}$ its ring of integers, $M_{k}$ the set of absolute values of $k, M_{k}^{0}$ all non-archimedian places, and $M_{k}^{\infty}$ archimedian places of $M_{k}$.

For any two elements $\alpha, \beta \in \mathcal{O}_{k}$ the greatest common divisor is defined as

$$
\operatorname{gcd}(\alpha, \beta)=\prod_{p \in \mathcal{O}_{k}} p^{\min \left\{\nu_{p}(\alpha), \nu_{p}(\beta)\right\}},
$$

where $\nu_{p}$ is the valuation corresponding to the prime $p$; see [20] for details. The logarithmic gcd is

$$
\log \operatorname{gcd}(\alpha, \beta)=\sum_{\nu \in M_{k}^{0}} \min \{\nu(\alpha), v(\beta)\}
$$

For a valuation $\nu \in M_{k}$, define

$$
\begin{aligned}
\nu^{+}: k & \longrightarrow[0, \infty], \\
\alpha & \longrightarrow \max \{v(\alpha), 0\} .
\end{aligned}
$$

The generalized logarithmic greatest common divisor of two elements $\alpha, \beta \in$ $k$ is defined as

$$
h_{g c d}(\alpha, \beta)=\sum_{\nu \in M_{k}} \min \left\{\nu^{+}(\alpha), \nu^{+}(\beta)\right\} .
$$

Notice that $\nu^{+}$can be viewed as a height function on $\mathbb{P}^{1}(k)=k \cup\{\infty\}$, where we set $\nu^{+}(\infty)=0$. This leads to the generalized logarithmic greatest common divisor being viewed also as a height function:

$$
\begin{aligned}
G_{\nu}: \mathbb{P}^{1} \times \mathbb{P}^{1} & \rightarrow[0, \infty] \\
(\alpha, \beta) & \rightarrow \min \left\{\nu^{+}(\alpha), \nu^{+}(\beta)\right\}
\end{aligned}
$$

In view of the above we have

$$
h_{g c d}(\alpha, \beta)=\sum_{\nu \in M_{k}} G_{\nu}
$$


In 20] it was given a theoretical interpretation of the function $G_{\nu}$ in terms of blowups.

Lemma 4. For a weighted integer tuple $\mathbf{x}=\left(x_{0}, \ldots, x_{n}\right) \in \mathcal{O}_{k}^{n+1}$ the weighted greatest common divisor is given by

$$
\operatorname{wgcd}_{\mathfrak{w}}(\mathbf{x})=\prod_{p \in \mathcal{O}_{k}} p^{\min \left\{\left\lfloor\frac{\nu_{p}\left(x_{0}\right)}{q_{0}}\right\rfloor, \ldots,\left\lfloor\frac{\nu_{p}\left(x_{n}\right)}{q_{n}}\right\rfloor\right\}}
$$

Proof. The proof is elementary. From Prop. 1 we have that

$$
\operatorname{wgcd}_{\mathfrak{w}}(\mathbf{x})=\prod_{j=1}^{t} p_{j}^{\alpha_{j}}
$$

where $\alpha_{i, j}=\min \left\{\left\lfloor\frac{\alpha_{j, i}}{q_{i}}\right\rfloor \mid i=0, \ldots, n\right\}$, for each $j=1, \ldots, t$. But $\alpha_{j, i}=\nu_{p}\left(x_{i}\right)$, for each $i=0, \ldots, n$. The rest follows.

As above, the logarithmic weighted greatest common divisor is

$$
\log \operatorname{wgcd}_{\mathfrak{w}}(\mathbf{x})=\sum_{\nu \in M_{k}^{0}} \min \left\{\left\lfloor\frac{\nu_{p}\left(x_{0}\right)}{q_{0}}\right\rfloor, \ldots,\left\lfloor\frac{\nu_{p}\left(x_{n}\right)}{q_{n}}\right\rfloor\right\}
$$

Consider now $\mathbf{x}=\left(x_{0}, \ldots, x_{n}\right) \in k^{n+1}$ with weights $\mathfrak{w}=\left(q_{0}, \ldots, q_{n}\right)$. The generalized weighted greatest common divisor is defined as follows

$$
\mathrm{h}_{\mathrm{wgcd}}(\mathbf{x})=\prod_{p \in \mathcal{O}_{k}} p^{\min \left\{\left\lfloor\frac{\nu_{p}^{+}\left(x_{0}\right)}{q_{0}}\right\rfloor, \ldots,\left\lfloor\frac{\nu_{p}^{+}\left(x_{n}\right)}{q_{n}}\right\rfloor\right\}}
$$

and the logarithmic weighted greatest common divisor is

$$
\log \mathrm{h}_{\mathrm{wgcd}}(\mathbf{x})=\sum_{\nu \in M_{k}^{0}} \min \left\{\left\lfloor\frac{\nu_{p}^{+}\left(x_{0}\right)}{q_{0}}\right\rfloor, \ldots,\left\lfloor\frac{\nu_{p}^{+}\left(x_{n}\right)}{q_{n}}\right\rfloor\right\}
$$

Let $q=\operatorname{gcd}\left(q_{0}, \ldots, q_{n}\right)$ and $\bar{q}_{i}=\frac{q_{i}}{q}$. Hence, we get a new set of well-formed weights $\bar{q}=\left(\bar{q}_{0}, \bar{q}_{1}, \ldots, \bar{q}_{n}\right)$.

The factorization of coordinates of $\mathbf{x}$ into primes is $x_{i}=\prod_{p \in \mathcal{O}_{k}} p^{\nu_{p}\left(x_{i}\right)}$, for $i=0, \ldots, n$. Then we have:

Lemma 5. The absolute weighted greatest common divisor is

$$
\overline{\operatorname{wgcd}}(\mathbf{x})=\left(\prod_{p \in \mathcal{O}_{k}} p^{\min \left\{\left\lfloor\frac{\nu_{p}\left(x_{0}\right)}{\bar{q}_{0}}\right\rfloor, \ldots,\left\lfloor\frac{\nu_{p}\left(x_{n}\right)}{\bar{q}_{n}}\right\rfloor\right\}}\right)^{\frac{1}{q}}
$$

Proof. The proof is similar to the previous Lemma, but using Prop. 2 .

Accordingly we define the generalized absolute weighted greatest common divisor by

$$
\mathrm{h}_{\mathrm{wgcd}}(\mathbf{x})=\frac{1}{q} \prod_{p \in \mathcal{O}_{k}} p^{\min \left\{\left\lfloor\frac{\nu_{p}^{+}\left(x_{0}\right)}{\bar{q}_{0}}\right\rfloor, \ldots,\left\lfloor\frac{\nu_{p}^{+}\left(x_{n}\right)}{\bar{q}_{n}}\right\rfloor\right\}}
$$


and the logarithmic absolute weighted greatest common divisor is

$$
\log \mathrm{h}_{\mathrm{wgcd}}(\mathbf{x})=\sum_{\nu \in M_{k}^{0}} \min \left\{\left\lfloor\frac{\nu_{p}^{+}\left(x_{0}\right)}{\bar{q}_{0}}\right\rfloor, \ldots,\left\lfloor\frac{\nu_{p}^{+}\left(x_{n}\right)}{\bar{q}_{n}}\right\rfloor\right\}
$$

Let us see an example.

Example 4. Let $\mathfrak{w}=(2,4,6,10)$ and $\mathfrak{p} \in \mathbb{W}_{\mathfrak{w}}^{3}(\mathbb{Q})$ such that

$$
\mathfrak{p}=\left[2^{3} \cdot 3^{2} \cdot 7^{3} ; 2^{5} \cdot 3^{7} \cdot 7 ; 2^{7} \cdot 3^{7} \cdot 7^{3} ; 2^{11} \cdot 3^{13} \cdot 7^{5}\right] .
$$

Then $\operatorname{wgcd}(\mathfrak{p})=2 \cdot 3$ and $\overline{\operatorname{wgcd}}(\mathfrak{p})=2 \cdot 3$. The normalized point is

$$
\overline{\mathfrak{p}}=\left[2 \cdot 7^{3} ; 2 \cdot 3^{3} \cdot 7 ; 2 \cdot 3 \cdot 7^{3} ; 2 \cdot 3^{3} \cdot 7^{5}\right]
$$

Using the previous two lemmas we have

$$
\operatorname{wgcd}(\mathfrak{p})=2^{\alpha_{1}} \cdot 3^{\alpha_{2}} \cdot 7^{\alpha_{3}}=2 \cdot 3 \cdot 7^{0}=2 \cdot 3 .
$$

where

$$
\begin{gathered}
\alpha_{1}=\min \left\{\left\lfloor\frac{3}{2}\right\rfloor,\left\lfloor\frac{5}{4}\right\rfloor,\left\lfloor\frac{7}{6}\right\rfloor,\left\lfloor\frac{11}{10}\right\rfloor\right\}, \alpha_{2}=\min \left\{\left\lfloor\frac{2}{2}\right\rfloor,\left\lfloor\frac{7}{4}\right\rfloor,\left\lfloor\frac{7}{6}\right\rfloor,\left\lfloor\frac{13}{10}\right\rfloor\right\}, \\
\alpha_{3}=\min \left\{\left\lfloor\frac{3}{2}\right\rfloor,\left\lfloor\frac{1}{4}\right\rfloor,\left\lfloor\frac{3}{6}\right\rfloor,\left\lfloor\frac{5}{10}\right\rfloor\right\}
\end{gathered}
$$

Similarly for $\overline{\mathrm{wgcd}}$ we have

$$
\overline{\operatorname{wgcd}}(\mathfrak{p})=\left(2^{\beta_{1}} \cdot 3^{\beta_{2}} \cdot 7^{\beta_{3}}\right)^{\frac{1}{2}}=\left(2^{2} \cdot 3^{2} \cdot 7^{0}\right)^{\frac{1}{2}}=2 \cdot 3,
$$

where

$$
\begin{gathered}
\beta_{1}=\min \left\{\left\lfloor\frac{3}{1}\right\rfloor,\left\lfloor\frac{5}{2}\right\rfloor,\left\lfloor\frac{7}{3}\right\rfloor,\left\lfloor\frac{11}{5}\right\rfloor\right\}, \beta_{2}=\min \left\{\left\lfloor\frac{2}{1}\right\rfloor,\left\lfloor\frac{7}{2}\right\rfloor,\left\lfloor\frac{7}{3}\right\rfloor,\left\lfloor\frac{13}{5}\right\rfloor\right\} \\
\beta_{3}=\min \left\{\left\lfloor\frac{3}{1}\right\rfloor,\left\lfloor\frac{1}{2}\right\rfloor,\left\lfloor\frac{3}{3}\right\rfloor,\left\lfloor\frac{5}{5}\right\rfloor\right\}
\end{gathered}
$$

Of course this is no surprise sine the normalization $\mathfrak{p}$ can be seen easily that is absolutely normalized.

\section{Normalized Points in Weighted PROJECTIVE SPACES}

Let $K$ be a field and $\left(q_{0}, \ldots, q_{n}\right) \in \mathbb{Z}^{n+1}$ a fixed tuple of positive integers called weights. Consider the action of $K^{\star}=K \backslash\{0\}$ on $\mathbb{A}^{n+1}(K)$ as follows

$$
\lambda \star\left(x_{0}, \ldots, x_{n}\right)=\left(\lambda^{q_{0}} x_{0}, \ldots, \lambda^{q_{n}} x_{n}\right)
$$

for $\lambda \in K^{*}$. The quotient of this action is called a weighted projective space and denoted by $\mathbb{W P}_{\left(q_{0}, \ldots, q_{n}\right)}^{n}(K)$. The space $\mathbb{W P}_{(1, \ldots, 1)}(K)$ is the usual projective space. The space $\mathbb{W} \mathbb{P}_{w}^{n}$ is called well-formed if

$$
\operatorname{gcd}\left(q_{0}, \ldots, \hat{q}_{i}, \ldots, q_{n}\right)=1, \quad \text { for each } i=0, \ldots, n .
$$

While most of the papers on weighted projective spaces are on well-formed spaces, we do not assume that here. We will denote a point $\mathfrak{p} \in \mathbb{W}_{w}^{n}(K)$ by $\mathfrak{p}=\left[x_{0}: x_{1}\right.$ : $\left.\cdots: x_{n}\right]$.

Weighted projective spaces are interesting since we can present a non singular algebraic variety as a hypersurface in a weighted projective space and deal with it as it would be a nonsingular hypersurface in a weighted projective space. For more on weighted projective spaces one can check [1, [6], [4, [9] among many others. 
In projective spaces, by means of the Veronese embedding, we could embed the same variety in different projective spaces. It turns out that we can do the same for varieties embedded in weighted projective spaces.

As above we let $k$ be a field. Let $R=\oplus_{i \geq 0} R_{i}$ be a graded ring. We further assume that

(i) $R_{0}=k$ is the ground field

(ii) $R$ is finitely generated as a ring over $k$

(iii) $R$ is an integral domain

Consider the polynomial ring $k\left[x_{0}, \ldots, x_{n}\right]$ where each $x_{i}$ has weight wt $x_{i}=q_{i}$. Every polynomial is a sum of monomials $x^{m}=\prod x_{i}^{m_{i}}$ with weight $\operatorname{wt}\left(x^{m}\right)=$ $\sum m_{i} q_{i}$. A polynomial $f$ is weighted homogenous of weight $m$ if every monomial of $f$ has weight $m$.

An ideal in a graded ring $I \subset R$ is called graded or weighted homogenous if $I=\oplus_{n \geq 0} I_{n}$, where $I_{n}=I \cap R_{n}$. Hence, $R=k\left[x_{0}, \ldots, x_{n}\right] / I$, where deg $x_{i}=q_{i}$ and $I$ is a homogenous prime ideal.

To the prime ideal $I$ corresponds an irreducible affine variety $C X=\operatorname{Spec} R=$ $V_{a}(I) \subset \mathbb{A}^{n+1}$.

Definition 1. A polynomial $f\left(x_{0}, \ldots, x_{n}\right)$ is called weighted homogenous of degree $d$ if it satisfies the following

$$
f\left(\lambda^{q_{0}} x_{0}, \lambda^{q_{1}} x_{1}, \ldots, \lambda^{q_{n}} x_{n}\right)=\lambda^{d} f\left(x_{0}, \ldots, x_{n}\right) .
$$

Let us consider a simple example of weighted homogenous polynomials.

Example 5. Let us consider a binary weighted form with weighted degree d and let $w=\left(q_{0}, q_{1}\right)$ be respectively the weights of $x_{0}$ and $x_{1}$. Then

$$
f\left(x_{0}, x_{1}\right)=\sum_{d_{0}, d_{1}} a_{d_{0}, d_{1}} x_{0}^{d_{0}} x_{1}^{d_{1}}, \text { such that } d_{0} q_{0}+d_{1} q_{1}=d
$$

and in decreasing powers of $x_{0}$ we have

$$
f\left(x_{0}, x_{1}\right)=a_{d / q_{0}, 0} x_{0}^{d / q_{0}}+\cdots+a_{d_{0}, d_{1}} x_{0}^{d_{0}} x_{1}^{d_{1}}+\cdots+a_{0, d / q_{1}} x_{1}^{d / q_{1}}
$$

By dividing this polynomial with $x_{1}^{d / q_{1}}$ and making a change of coordinates $X=$ $x_{0}^{q_{1}} / x_{1}^{q_{0}}$ we get

$$
\begin{aligned}
f\left(x_{0}, x_{1}\right) & =a_{d / q_{0}, 0} x_{0}^{d / q_{0}}+\cdots+a_{d_{0}, d_{1}} x_{0}^{d_{0}} x_{1}^{d_{1}}+\cdots+a_{0, d / q_{1}} x_{1}^{d / q_{1}} \\
& =a_{d / q_{0}, 0} \frac{x_{0}^{d / q_{0}}}{x_{1}^{d / q_{1}}}+\cdots+a_{d_{0}, d_{1}} \frac{x_{0}^{d_{0}} x_{1}^{d_{1}}}{x_{1}^{d / q_{1}}}+\cdots+a_{0, d / q_{1}} \\
& =a_{d / q_{0}, 0} X^{d / q_{0} q_{1}}+\cdots+a_{d_{0}, d_{1}} X^{d_{0} / q_{1}}+\cdots+a_{0, d / q_{1}}=f(X)
\end{aligned}
$$

Notice that the condition $f(P)=0$ is defined on the equivalence classes of Eq. (8). We define the quotient $V_{a}(I) \backslash\{0\}$ by the above equivalence by $V_{h}(I)$, where $h$ stands for homogenous. Then, we denote $X=\operatorname{Proj} R=V_{h}(I) \subset \mathbb{W} \mathbb{P}_{\mathfrak{w}}^{n}(k)$. It is a projective variety. Notice that $C X$ above is the affine cone over the projective variety $V_{h}(I)$.

Next we will define truncated rings and see the role that they play in the Veronese embedding. Define the $d$ 'th truncated ring $R^{[d]} \subset R$ by

$$
R^{[d]}=\bigoplus_{d \mid n} R_{n}=\bigoplus_{i \geq 0} R_{d i}
$$


Hence, $R^{[d]}$ is a graded ring and the elements have degree $d i$ in $R$ and degree $i$ in $R^{[d]}$. If $R$ is a graded ring then its subring $R^{[d]}$ is called the $d$-th Veronese subring.

Example 6. Let $R=k[x, y]$ with $w t(x)=w t(y)=1$. Then,

$$
R^{[2]}=\bigoplus_{i \geq 0} R_{2 i}=\bigoplus_{i \geq 0}\{f(x, y) \in k[x, y] \mid \operatorname{deg}(f)=2 i\} .
$$

Notice that the even degree polynomials in $k[x, y]$ are generated by $x^{2}, x y$, and $y^{2}$ hence we have that

$$
R^{[2]}=k\left[x^{2}, x y, y^{2}\right] \cong k[u, v, w] /\left\langle u w-v^{2}\right\rangle
$$

Now, if we consider the projective spaces we have that

$$
\operatorname{Proj}(k[x, y])=\mathbb{P}_{(1,1)}=\mathbb{P}^{1}
$$

while

$$
\operatorname{Proj}\left(k[u, v, w] /\left\langle u w-v^{2}\right\rangle\right)=V\left(u w-v^{2}\right) \subseteq \mathbb{P}_{(1,1,1)}=\mathbb{P}^{2}
$$

Hence we have that,

$$
\mathbb{P}^{1}(k)=\operatorname{Proj}(k[x, y]) \cong \operatorname{Proj}\left(k[x, y]^{2}\right) \subseteq \mathbb{P}^{2}(k) .
$$

This is exactly the degree-2 Veronese embedding of $\mathbb{P}^{1}(k) \hookrightarrow \mathbb{P}^{2}(k)$. The truncation of graded rings in this case corresponds to the degree-2 Veronese embedding.

The proof of the following lemma can be found in 9.

Lemma 6. Let $R$ be a graded ring and $d \in \mathbb{N}$. Then,

$$
\operatorname{Proj} R \cong \operatorname{Proj} R^{[d]}
$$

For some large enough $N$ and using the above Lem. 6 we can embed a weighted projective space $\mathbb{W} \mathbb{P}_{w}$ into a "straight" projective space $\mathbb{P}^{N}$.

Proposition 3. Consider the weighted polynomial ring $R=k\left[x_{0}, \ldots, x_{n}\right]$, where $q_{0}, \ldots, q_{n}$ are positive integers such that the weight of $x_{i}$ is $q_{i}$ and $d=\operatorname{gcd}\left(q_{0}, \ldots, q_{n}\right)$. The following are true:

i) $R^{[d]}=R$. Thus,

$$
\mathbb{W P}_{\left(q_{0}, \ldots, q_{n}\right)}^{n}(R)=\mathbb{W P}_{\left(\frac{q_{0}}{d}, \ldots, \frac{q_{n}}{d}\right)}^{n}(R) .
$$

ii) Suppose that $q_{0}, \ldots, q_{n}$ have no common factor, and that $d$ is a common factor of all $a_{i}$ for $i \neq j$ (and therefore coprime to $a_{j}$ ). Then the $d$ 'th truncation of $R$ is the polynomial ring

$$
R^{[d]}=k\left[x_{0}, \ldots, x_{j-1}, x_{j}^{d}, x_{j+1}, \ldots, x_{n}\right] .
$$

Thus, in this case

$$
\mathbb{W P}_{\left(q_{0}, \ldots, q_{n}\right)}^{n}(R)=\mathbb{W P}_{\left(\frac{q_{0}}{d}, \ldots, \frac{q_{j-1}}{d}, q_{j}, \frac{q_{j+1}}{d}, \ldots, \frac{q_{n}}{d}\right)}^{n}\left(R^{[d]}\right) .
$$

In particular by passing to a truncation $R^{[d]}$ of $R$ which is a polynomial ring generated by pure powers of $x_{i}$, we can always write any weighted projective space as a well formed weighted projective space. 
Proof. i) If $d \mid q_{i}$ for all $i=0, \ldots, n$ then the degree of every monomial is divisible by $d$ and so part i) is obvious. Hence, the truncation does not change anything.

ii) Since $d \mid q_{i}$ for every $i \neq j$ then $x_{i} \in \mathbb{R}^{[d]}$ for every $i \neq j$. But the only way that $x_{j}$ can occur in a monomial with degree divisible by $d$ is as a $d$ 'th power. Given

$$
R=k\left[x_{0}, \ldots, x_{j}, \ldots, x_{n}\right]
$$

then

and

$$
R^{[d]}=k\left[x_{0}, \ldots, x_{j}^{d}, \ldots, x_{n}\right]
$$

$$
\begin{aligned}
\mathbb{W P}_{\left(q_{0}, \ldots, q_{n}\right)}^{n}(R) & =\operatorname{Proj} k_{w}\left[x_{0}, \ldots, x_{j}, \ldots, x_{n}\right] \equiv \operatorname{Proj} k_{w / d}\left[x_{0}, \ldots, x_{j}^{d}, \ldots, x_{n}\right] \\
& =\mathbb{W P}_{\left(\frac{q_{0}}{d}, \ldots, \frac{q_{j-1}}{d}, q_{j}, \frac{q_{j+1}}{d}, \ldots, \frac{q_{n}}{d}\right)}^{n}\left(R^{[d]}\right) .
\end{aligned}
$$

This completes the proof.

Hence, the above result shows that any weighted projective space is isomorphic to a well formed weighted projective space.

For the rest of this paper we will always assume that $R$ is the ring of integers $\mathcal{O}_{k}$ for some number field $k$. We will call a point $\mathfrak{p} \in \mathcal{O}_{k}^{n}$ a normalized point if the weighted greatest common divisor of its coordinates is 1 . Similarly an absolutely normalized point is called a point $\mathfrak{p}$ such that $\overline{\operatorname{wgcd}}(\mathfrak{p})=1$.

Lemma 7. Let $\mathfrak{w}=\left(q_{0}, \ldots, q_{n}\right)$ be a set of weights and $q=\operatorname{gcd}\left(q_{0}, \ldots, q_{n}\right)$. For any point $\mathfrak{p} \in \mathbb{W P}_{w}^{n}(k)$, there exists its normalization given by

$$
\mathfrak{q}=\frac{1}{\operatorname{wgcd}(\mathfrak{p})} \star \mathfrak{p} .
$$

Moreover, this normalization is unique up to a multiplication by a q-root of unity.

Proof. Let $\mathfrak{p}=\left[x_{0}: \ldots, x_{n}\right] \in \mathbb{W}_{\mathbb{P}}^{n}(k)$ and $\mathfrak{p}_{1}=\left[\alpha_{0}: \cdots: \alpha_{n}\right]$ and $\mathfrak{p}_{2}=\left[\beta_{0}: \cdots\right.$ : $\left.\beta_{n}\right]$ two different normalizations of $\mathfrak{p}$. Then exists non-zero $\lambda_{1}, \lambda_{2} \in k$ such that

$$
\mathfrak{p}=\lambda_{1} \star \mathfrak{p}_{1}=\lambda_{2} \star \mathfrak{p}_{2},
$$

or in other words

$$
\left(x_{0}, \ldots, x_{n}\right)=\left(\lambda_{1}^{q_{0}} \alpha_{0}, \ldots, \lambda_{1}^{q_{i}} \alpha_{i}, \ldots\right)=\left(\lambda_{2}^{q_{0}} \beta_{0}, \ldots, \lambda_{2}^{q_{i}} \beta_{i}, \ldots\right) .
$$

Thus,

$$
\left(\alpha_{0}, \ldots, \alpha_{i}, \ldots, \alpha_{n}\right)=\left(r^{q_{0}} \beta_{0}, \ldots, r^{q_{i}} \beta_{i}, \ldots, r^{q_{n}} \beta_{n}\right) .
$$

for $r=\frac{\lambda_{2}}{\lambda_{1}} \in k$. Thus, $r^{q_{i}}=1$ for all $i=0, \ldots, n$. Therefore, $r^{q}=1$. This completes the proof.

Thus we have the following:

Corollary 1. Points in a well-formed weighted projective space $\mathbb{W}_{w}^{n}(k)$ have unique normalizations.

Here is an example which illustrates Lem. 7

Example 7. Let $\mathfrak{p}=\left[x_{0}, x_{1}, x_{2}, x_{3}\right] \in \mathbb{W}_{(2,4,6,10)}^{3}(\mathbb{Q})$ be a normalized point. Hence,

$$
\operatorname{wgcd}\left(x_{0}, x_{1}, x_{2}, x_{3}\right)=1 \text {. }
$$

Since $q=\operatorname{gcd}(2,4,6,10)=2$, then we can take $r$ such that $r^{2}=1$. Hence, $r= \pm 1$. Therefore, the point

$$
(-1) \star \mathfrak{p}=\left[-x_{0}: x_{1}:-x_{2}:-x_{3}\right]
$$


is also normalized.

However, if $\mathfrak{p}=\left[x_{0}, x_{1}, x_{2}, x_{3}\right] \in \mathbb{W}_{(1,2,3,5)}^{3}(\mathbb{Q})$ is normalized then it is unique, unless some of the coordinates are zero. For example the points $[0,1,0,0]$ and $[0,-1,0,0]$ are equivalent and both normalized.

Thus, the weighted greatest common divisor gives us a very nice and efficient way to represent point in weighted moduli spaces via normalized points. Such normalized points have as small coefficients as possible. We define the magnitude or naive height of a point $\mathfrak{p} \in \mathbb{W P}_{w}^{n}(k)$ as

$$
\mathfrak{s}(\mathfrak{p})=\max \left\{\left|x_{0}\right|_{\infty}^{\frac{1}{q_{0}}}, \ldots,\left|x_{n}\right|_{\infty}^{\frac{1}{q_{n}}}\right\}
$$

where $x_{0}, \ldots, x_{i}$ are the coordinates of the normalized point.

Lemma 8. Let $\mathfrak{w}$ be a set of weights, $k$ a number field, and $\mathbb{W P}_{\mathfrak{w}}^{n}(k)$ a well-formed weighted projective space. Then the function

$$
\mathfrak{s}: \mathbb{W} \mathbb{P}_{\mathfrak{w}}^{n}(k) \rightarrow \mathbb{R}
$$

is well defined.

Proof. Since $\mathbb{W P}_{\mathfrak{w}}^{n}(k)$ is well-formed then from Cor. 1 for each point $\mathfrak{p} \in \mathbb{W}_{\mathfrak{w}}^{n}(k)$ its normalization is unique. The rest follows.

The above function provides a nice way to order points in $\mathbb{W}_{\mathfrak{w}}^{n}(k)$. Moreover, each point in a well-formed space $\mathbb{W P}_{\mathfrak{w}}^{n}(k)$ is now uniquely represented with "small" coefficients. This idea, first suggested in [16] was explored in [3] and [2] to create a database and hyperelliptic curves of genus $g=2,3$.

Of course, the values of $\mathfrak{s}$ change as the field is extended. We see an example below.

Example 8. Let $\mathfrak{w}=(2,3,5)$ and $\mathfrak{p}=[7: 0: 0] \in \mathbb{W}_{\mathfrak{w}}^{2}(\mathbb{Q})$. Then $\operatorname{wgcd}_{\mathbb{Q}}(\mathfrak{p})=1$ and its normalization is $\overline{\mathfrak{p}}=\mathfrak{p}$. Hence, $\mathfrak{s}_{\mathbb{Q}}(\mathfrak{p})=\sqrt{7}$.

Consider now the field $K=\mathbb{Q}(\sqrt{7})$ and the same point $\mathfrak{p}=[7: 0: 0] \in \mathbb{W}_{\mathfrak{w}}^{2}(K)$. Then $\operatorname{wgcd}_{K}(\mathfrak{p})=\sqrt{7}$ and the normalization of $\mathfrak{p}$ is $\overline{\mathfrak{p}}=[1: 0: 0]$. Hence, $\mathfrak{s}_{K}(\mathfrak{p})=1$.

So a different measuring of the size of points in $\mathbb{W}_{\mathfrak{w}}^{n}(k)$ is needed which behaves similarly to a height function on the regular projective space $\mathbb{P}^{n}(k)$. We explore this in the next section.

\section{Heights on the Weighted PRojective space}

In our attempt to define a height on the weighted projective space we fix the following notation for the rest of the paper.

$k$ is a number field.

$\mathcal{O}_{k}$ ring of integers of $k$.

$M_{k} \quad$ a complete set of absolute values of $k$

$M_{k}^{0} \quad$ the set of all non-archimedian places in $M_{k}$

$M_{k}^{\infty}$ the set of archimedian places 
$\mathcal{X} / k \quad$ a smooth projective variety defined over $k$.

Let $k$ be a given number field, $\mathcal{O}_{k}$ its ring of integers, and $M_{k}$ the set of absolute values on $k$. For a place $\nu \in M_{k}$, the corresponding absolute value is denoted by $|\cdot|_{\nu}$, normalized with respect to $k$ such that the product formula holds and the Weil height is

$$
H(x)=\prod_{\nu} \max \left\{1,|x|_{\nu}\right\}
$$

For a point $\mathbf{x} \in k^{n+1}$ and a place $\nu \in M_{k}$ we define $|\mathbf{x}|_{\nu}=\max _{i}\left|x_{i}\right|_{\nu}$. For $\mathbf{x}=\left(x_{0}: \cdots: x_{n}\right) \in \mathbb{P}^{n}(k)$ we have the height of $\mathbf{x}$ defined as

$$
H(\mathbf{x})=\prod_{\nu} \max \left\{\left|x_{0}\right|_{\nu}, \ldots,\left|x_{n}\right|_{\nu}\right\}=\prod_{\nu}|x|_{\nu}
$$

Because of the product formula, the height of $\mathbf{x}$ is well defined.

Let $k$ be an algebraic number field and $[k: \mathbb{Q}]=n$. With $M_{k}$ we will denote the set of all absolute values in $K$. For $v \in M_{k}$, the local degree at $v$, denoted $n_{v}$ is

$$
n_{v}=\left[k_{v}: \mathbb{Q}_{v}\right]
$$

where $K_{v}, \mathbb{Q}_{v}$ are the completions with respect to $v$. Let $L / k$ be an extension of number fields, and let $v \in M_{k}$ be an absolute value on $k$. Then

$$
\sum_{\substack{w \in M_{L} \\ w \mid v}}\left[L_{w}: k_{v}\right]=[L: k]
$$

is known as the degree formula. For $x \in k^{\star}$ we have the product formula

$$
\prod_{v \in M_{k}}|x|_{v}^{n_{v}}=1
$$

Given a point $\mathfrak{p} \in \mathbb{P}^{n}(\overline{\mathbb{Q}})$ with $\mathfrak{p}=\left[x_{0}, \ldots, x_{n}\right]$, the field of definition of $\mathfrak{p}$ is

$$
\mathbb{Q}(\mathfrak{p})=\mathbb{Q}\left(\frac{x_{0}}{x_{j}}, \ldots, \frac{x_{n}}{x_{j}}\right)
$$

for any $j$ such that $x_{j} \neq 0$. Next we try to generalize some of these concepts for the weighted projective spaces $\mathbb{W P}_{\mathfrak{w}}(k)$.

Let $\mathfrak{w}=\left(q_{0}, \ldots, q_{n}\right)$ be a set of heights and $\mathbb{W} \mathbb{P}^{n}(k)$ the weighted projective space over a number field $k$. Let $\mathfrak{p} \in \mathbb{W P}^{n}(k)$ a point such that $\mathfrak{p}=\left[x_{0}, \ldots, x_{n}\right]$. Without any loss of generality we can assume that $\mathfrak{p}$ is normalized.

The field of absolute normalization of $\mathfrak{p}$ is defined as $\mathbb{Q}(\overline{\operatorname{wgcd}}(\mathfrak{p}))$.

Definition 2. Let $\mathfrak{w}=\left(q_{0}, \ldots, q_{n}\right)$ be a set of heights and $\mathbb{W P}^{n}(k)$ the weighted projective space over a number field $k$. Let $\mathfrak{p} \in \mathbb{W}^{n}(k)$ a point such that $\mathfrak{p}=$ $\left[x_{0}, \ldots, x_{n}\right]$. We define the weighted multiplicative height of $P$ as

$$
\mathfrak{h}_{k}(\mathfrak{p}):=\prod_{v \in M_{k}} \max \left\{\left|x_{0}\right|_{v}^{\frac{n_{v}}{q_{0}}}, \ldots,\left|x_{n}\right|_{v}^{\frac{n_{v}}{q_{n}}}\right\}
$$

The logarithmic height of the point $\mathfrak{p}$ is defined as follows

$$
\mathfrak{h}_{k}^{\prime}(\mathfrak{p}):=\log \mathfrak{h}_{k}(\mathfrak{p})=\sum_{v \in M_{k}} \max _{0 \leq j \leq n}\left\{\frac{n_{v}}{q_{j}} \cdot \log \left|x_{j}\right|_{v}\right\} .
$$

Next we will give some basic properties of heights functions. 
Proposition 4. Let $k$ be a number field and $\mathfrak{p} \in \mathbb{W} \mathbb{P}^{n}(k)$ with weights $w=$ $\left(q_{0}, \ldots, q_{n}\right)$. Then the following are true:

i) The height $\mathfrak{h}_{k}(\mathfrak{p})$ is well defined, in other words it does not depend on the choice of coordinates of $\mathfrak{p}$

ii) $\mathfrak{h}_{k}(\mathfrak{p}) \geq 1$.

Proof. i) Let $\mathfrak{p}=\left[x_{0}, \ldots, x_{n}\right] \in \mathbb{W P}^{n}(k)$. Since $\mathfrak{p}$ is a point in the weighted projective space, any other choice of homogenous coordinates for $\mathfrak{p}$ has the form $\left[\lambda^{q_{0}} x_{0}, \ldots, \lambda^{q_{n}} x_{n}\right]$, where $\lambda \in k^{*}$. Then

$$
\begin{aligned}
\mathfrak{h}_{k}\left(\left[\lambda^{q_{0}} x_{0}, \ldots, \lambda^{q_{n}} x_{n}\right]\right) & =\prod_{v \in M_{k}} \max _{0 \leq i \leq n}\left\{\left|\lambda^{q_{i}} x_{i}\right|_{v}^{n_{v} / q_{i}}\right\} \\
& =\prod_{v \in M_{k}}|\lambda|_{v}^{n_{v}} \max _{0 \leq i \leq n}\left\{\left|x_{i}\right|_{v}^{n_{v} / q_{i}}\right\} \\
& =\left(\prod_{v \in M_{k}}|\lambda|_{v}^{n_{v}}\right) \cdot\left(\prod_{v \in M_{k}} \max _{0 \leq i \leq n}\left\{\left|x_{i}\right|_{v}^{n_{v} / q_{i}}\right\}\right)
\end{aligned}
$$

Applying the product formula we have

$$
\mathfrak{h}_{K}\left(\left[\lambda_{0}^{q} x_{0}, \ldots, \lambda_{n}^{q} x_{n}\right]\right)=\prod_{v \in M_{K}} \max _{0 \leq i \leq n}\left\{\left|x_{i}\right|_{v}^{n_{v} / q_{i}}\right\}=\mathfrak{h}_{K}(\mathfrak{p})
$$

This completes the proof of the first part.

ii) For every point $\mathfrak{p} \in \mathbb{W} \mathbb{P}^{n}(k)$ we can find a representative $\mathfrak{p}^{\prime}$ of $\mathfrak{p}$ with weighted homogenous coordinates such that one of the coordinates is 1 . Assume, that $\mathfrak{p}=$ $\left[x_{0}: \ldots: x_{i}: \cdots: x_{n}\right]$ such that $x_{i} \neq 0$. Then take $\mathfrak{p}^{\prime}=\lambda \star \mathfrak{p}$, where $\lambda=\left(\frac{1}{x_{i}}\right)^{\frac{1}{q_{i}}}$ and $\mathfrak{p}^{\prime}=\left[y_{0}: \ldots: 1: \cdots: y_{n}\right]$, where

$$
y_{j}=x_{j} \cdot x_{i}^{-\frac{q_{j}}{q_{i}}}
$$

for $j=0, \ldots, n$ and $j \neq i$. The the height is

$$
\begin{aligned}
\mathfrak{h}_{k}\left(\mathfrak{p}^{\prime}\right) & =\prod_{v \in M_{k}} \max \left\{\left|x_{0}\right|_{v}^{n_{v} / q_{0}}, \ldots,\left|x_{n}\right|_{v}^{n_{v} / q_{n}}\right\} \\
& =\prod_{v \in M_{k}} \max \left\{1,\left|y_{0}\right|_{v}^{n_{v} / q_{0}}, \ldots,\left|y_{n}\right|_{v}^{n_{v} / q_{n}}\right\} .
\end{aligned}
$$

Hence, every factor in the product is at least 1 . Therefore, $\mathfrak{h}_{K}(P) \geq 1$.

Let us see an example.

Example 9. Consider the set of weights $\mathfrak{w}=(2,3,5)$ and the point $\mathfrak{p}=[7: 0$ : $0] \in \mathbb{W} \mathbb{P}_{\mathfrak{w}}(\mathbb{Q})$. Then, $\operatorname{wgcd}_{\mathbb{Z}}(\mathfrak{p})=1$ and

$$
\begin{aligned}
\mathfrak{h}_{\mathbb{Q}}(\mathfrak{p}) & =\max \left\{\sqrt{|7|_{7}}, \sqrt[3]{|0|_{7}}, \sqrt[5]{|0|_{7}}\right\} \cdot \max \left\{\sqrt{|7|_{\infty}}, \sqrt[3]{|0|_{\infty}}, \sqrt[5]{|0|_{\infty}}\right\} \\
& =\max \left\{\sqrt{\frac{1}{7}}, 1,1\right\} \cdot \max \{\sqrt{7}, 1,1\}=\sqrt{7}
\end{aligned}
$$

Let us now consider $K=\mathbb{Q}(\sqrt{7})$. Then, $\operatorname{wgcd}_{\mathcal{O}_{K}}(\mathfrak{p})=\sqrt{7}$ and over $K$ we have

$$
\mathfrak{p}=\frac{1}{\sqrt{7}} \star[7: 0: 0]=[1: 0: 0]
$$


so $\mathfrak{h}_{K}(\mathfrak{p})=1$.

From the Definition 2 we see that $\mathfrak{s}_{k}$ ca be defined as

$$
\mathfrak{s}_{k}(\mathfrak{p})=\prod_{M_{k}^{\infty}} \max \left\{\left|x_{0}\right|_{v}^{\frac{n_{v}}{q_{0}}}, \ldots,\left|x_{n}\right|_{v}^{\frac{n_{v}}{q_{n}}}\right\}
$$

and

$$
\mathfrak{h}_{k}(\mathfrak{p})=\mathfrak{s}_{k}(\mathfrak{p}) \cdot \prod_{v \in M_{k}^{0}} \max \left\{\left|x_{0}\right|_{v}^{\frac{n_{v}}{q_{0}}}, \ldots,\left|x_{n}\right|_{v^{\frac{n_{v}}{q_{n}}}}\right\}
$$

Let $\mathfrak{w}, k$ be as above and $\mathfrak{p} \in \mathbb{W P}^{n}(k)$ such that $p=\left[x_{0}: x_{1}: \ldots: x_{n}\right]$. Denote by $K=k(\overline{\operatorname{wgcd}}(\mathfrak{p}))$. Then, over $K$, the weighted greatest common divisor is the same as the absolute greatest common divisor,

$$
\operatorname{wgcd}_{K}(\mathfrak{p})=\overline{\operatorname{wgcd}}_{K}(\mathfrak{p}) .
$$

Moreover, $[K: k]<\infty$ and we have the following.

Proposition 5. Let $\mathfrak{w}, K=\mathbb{Q}(\overline{\operatorname{wgcd}}(\mathfrak{p}))$, and $\mathfrak{p} \in \mathbb{W P}^{n}(K)$, say $\mathfrak{p}=\left[x_{0}: x_{1}\right.$ : $\left.\ldots: x_{n}\right]$. Then the following are true:

i) If $\mathfrak{p}$ is normalized in $K$, then

$$
\mathfrak{h}_{K}(\mathfrak{p})=\mathfrak{h}_{\infty}(\mathfrak{p})=\max _{0 \leq i \leq n}\left\{\left|x_{i}\right|_{\infty}^{n_{\nu} / q_{i}}\right\} .
$$

ii) If $L / K$ is a finite extension, then

$$
\mathfrak{h}_{L}(\mathfrak{p})=\mathfrak{h}_{K}(\mathfrak{p})^{[L: K]}
$$

Proof. Let $\mathfrak{p}=\left[x_{0}, \ldots, x_{n}\right] \in \mathbb{W P}^{n}(K)$. Then, $\mathfrak{p}$ will have a representative $\left[y_{0}, \ldots, y_{n}\right]$ such that $y_{i} \in \mathcal{O}_{K}$ for all $i=0, \ldots, n$ and $\operatorname{wgcd}\left(y_{0}, \ldots, y_{n}\right)=1$. With such representative for the coordinates of $\mathfrak{p}$, the non-Archimedean absolute values give no contribution to the height, and we obtain

$$
\mathfrak{h}_{K}(\mathfrak{p})=\max _{0 \leq j \leq n}\left\{\left|x_{j}\right|_{\infty}^{n_{\nu} / q_{j}}\right\}
$$

ii) Let $L$ be a finite extension of $k$ and $M_{L}$ the corresponding set of absolute values. Then,

$$
\begin{aligned}
\mathfrak{h}_{L}(\mathfrak{p}) & =\prod_{w \in M_{L}} \max _{0 \leq i \leq n}\left\{\left|x_{i}\right|_{w}^{n_{w} / q_{i}}\right\}=\prod_{v \in M_{k}} \prod_{\substack{w \in M_{L} \\
w \mid v}} \max _{0 \leq i \leq n}\left\{\left|x_{i}\right|_{v}^{n_{w} / q_{i}}\right\}, \quad\left(\text { since } x_{i} \in k\right) \\
& =\prod_{v \in M_{k}} \max _{0 \leq i \leq n}\left\{\left|x_{i}\right|_{v}^{\frac{n_{v} \cdot[L: K]}{q_{i}}}\right\}, \quad \text { (degree formula) } \\
& =\prod_{v \in M_{k}} \max _{0 \leq i \leq n}\left\{\left|x_{i}\right|_{v}^{n_{v} / q_{i}}\right\}^{[L: k]}=\mathfrak{h}_{k}(\mathfrak{p})^{[L: k]}
\end{aligned}
$$

This completes the proof.

Corollary 2. If $\mathfrak{p}$ is absolutely normalized over a number field $k$ then

$$
\mathfrak{h}_{k}(\mathfrak{p})=\mathfrak{h}_{\infty}(\mathfrak{p})=\mathfrak{s}_{k}(\mathfrak{p}) .
$$

The next example illustrates the previous Proposition. 
Example 10. Let $\mathfrak{w}=(2,4)$ and $\mathfrak{p}=\left[5 \cdot 3,5^{2} \cdot 7\right]$. Then $\operatorname{wgcd}\left(5 \cdot 3,5^{2} \cdot 7\right)=1$. The height $\mathfrak{h}_{\mathbb{Q}}(\mathfrak{p})$ is

$$
\begin{aligned}
\mathfrak{h}_{\mathbb{Q}}(\mathfrak{p}) & =\max \left\{\left(\frac{1}{5}\right)^{\frac{1}{2}},\left(\frac{1}{25}\right)^{\frac{1}{4}}\right\} \cdot \max \left\{\left(\frac{1}{3}\right)^{\frac{1}{2}}, 1\right\} \cdot \max \left\{1,\left(\frac{1}{7}\right)^{\frac{1}{4}}\right\} \cdot(5 \cdot 3)^{\frac{1}{2}} \\
& =\left(\frac{1}{5}\right)^{\frac{1}{2}} \cdot(5 \cdot 3)^{\frac{1}{2}}=\sqrt{3}
\end{aligned}
$$

The absolute weighted greatest common divisor is

$$
\overline{\operatorname{wgcd}}\left(5 \cdot 3,5^{2} \cdot 7\right)=\sqrt{5} .
$$

Let $K=\mathbb{Q}(\sqrt{5})$ and compute $\mathfrak{h}_{K}(\mathfrak{p})$. Over $K$ the point $\mathfrak{p}$ is $\mathfrak{p}=[3: 7]$. Then

$$
\mathfrak{h}_{K}(\mathfrak{p})=\max \left\{|3|_{\infty}^{2 / 2},|7|_{\infty}^{2 / 4}\right\}=\max \{3, \sqrt{7}\}=3,
$$

as expected from Prop. 5, ii).

Let $q=q_{0} q_{1} \cdots q_{n}$ and consider the map

$$
\begin{aligned}
\phi: \quad & \mathbb{W} \mathbb{P}^{n}(k) \rightarrow \mathbb{P}^{n}(k) \\
& {\left[x_{0}, \ldots, x_{n}\right] \rightarrow\left[x_{0}^{\frac{q}{q_{0}}}, \ldots, x_{n}^{\frac{q}{q_{n}}}\right] }
\end{aligned}
$$

Lemma 9. Given $\phi$ and $q$ satisfying the above conditions we have i) $\phi$ is well-defined

ii) $\mathfrak{h}_{\mathbb{Q}}(\mathfrak{p})=H_{\mathbb{Q}}(\phi(\mathfrak{p}))^{\frac{1}{q}}$

Proof. Let $\mathbf{x}=\left[x_{0}, \ldots, x_{n}\right]$ and $\mathbf{y}=\left[y_{0}, \ldots, y_{n}\right]$ be points in $\mathbb{W}^{n}$ such that $\mathbf{x}=$ $\lambda \star \mathbf{y}$. Then, $\mathbf{x}=\left[\lambda^{q_{0}} y_{0}, \ldots, \lambda^{q_{n}} y_{n}\right]$ and

$$
\phi(\mathbf{x})=\left[\lambda^{q} y_{0}^{\frac{q}{q_{0}}}, \ldots, \lambda^{q} y_{n}^{\frac{q}{q_{n}}}\right]=\phi(\mathbf{y}) .
$$

Let $\mathfrak{p}=\left[x_{0}, \ldots, x_{n}\right]$ and $\overline{\mathfrak{p}}=\phi(\mathfrak{p})$. For the second part, by definition we have that

$$
\mathfrak{h}(\mathfrak{p})=\prod_{\nu \in M_{k}} \max \left\{\left|x_{i}\right|_{\nu}^{\frac{n_{v}}{q_{i}}}\right\}
$$

and

$$
H(\phi(\mathfrak{p}))=\prod_{\nu \in M_{k}} \max \left\{\left|x_{i}^{\frac{q}{q_{i}}}\right|_{\nu}^{n_{v}}\right\} .
$$

Then, we have the following

$$
(H(\phi(\mathfrak{p})))^{1 / q}=\left(\prod_{\nu \in M_{k}} \max \left\{\left|x_{i}^{\frac{q}{q_{i}}}\right|_{\nu}^{n_{v}}\right\}\right)^{1 / q}=\prod_{\nu \in M_{k}} \max \left\{\left|x_{i}^{\frac{1}{q_{i}}}\right|_{\nu}^{n_{v}}\right\}=\mathfrak{h}(\mathfrak{p}) .
$$

Therefore, we get $\mathfrak{h}_{\mathbb{Q}}(\mathfrak{p})^{q}=H_{\mathbb{Q}}(\phi(\mathfrak{p}))$.

Corollary 3. The following holds for logarithmic heights

$$
q \log \mathfrak{h}(\mathfrak{p})=\log H(\phi(\mathfrak{p}) .
$$


Using Prop. 5. part ii), we can define the height on $\mathbb{W} \mathbb{P}^{n}(\overline{\mathbb{Q}})$. The height of a point on $\mathbb{W P}^{n}(\overline{\mathbb{Q}})$ is called the absolute (multiplicative) weighted height and is the function

$$
\begin{aligned}
\tilde{\mathfrak{h}}: \mathbb{W} \mathbb{P}^{n}(\overline{\mathbb{Q}}) & \rightarrow[1, \infty) \\
\tilde{\mathfrak{h}}(\mathfrak{p}) & =\mathfrak{h}_{K}(\mathfrak{p})^{1 /[K: \mathbb{Q}]},
\end{aligned}
$$

where $\mathfrak{p} \in \mathbb{W P}^{n}(K)$, for any $K$ which contains $\mathbb{Q}(\overline{\operatorname{wgcd}}(\mathfrak{p}))$. The absolute (logarithmic) weighted height on $\mathbb{W P}^{n}(\overline{\mathbb{Q}})$ is the function

$$
\begin{aligned}
\tilde{\mathfrak{h}}^{\prime}: \mathbb{W P}^{n}(\overline{\mathbb{Q}}) & \rightarrow[0, \infty) \\
\tilde{\mathfrak{h}}^{\prime}(\mathfrak{p}) & =\log \mathfrak{h}(\mathfrak{p})=\frac{1}{[K: \mathbb{Q}]} \tilde{\mathfrak{h}}_{K}(\mathfrak{p}) .
\end{aligned}
$$

Lemma 10. The height is invariant under Galois conjugation. In other words, for $\mathfrak{p} \in \mathbb{W P}^{n}(\overline{\mathbb{Q}})$ and $\sigma \in G_{\mathbb{Q}}$ we have $\mathfrak{h}\left(\mathfrak{p}^{\sigma}\right)=\mathfrak{h}(\mathfrak{p})$.

Proof. Let $\mathfrak{p}=\left[x_{0}, \ldots, x_{n}\right] \in \mathbb{W P}^{n}(\overline{\mathbb{Q}})$. Let $K$ be a finite Galois extension of $\mathbb{Q}$ such that $\mathfrak{p} \in \mathbb{W} \mathbb{P}^{n}(K)$. Let $\sigma \in G_{\mathbb{Q}}$. Then $\sigma$ gives an isomorphism

$$
\sigma: K \rightarrow K^{\sigma}
$$

and also identifies the sets $M_{K}$, and $M_{K^{\sigma}}$ as follows

$$
\begin{aligned}
\sigma: M_{K} & \rightarrow M_{K^{\sigma}} \\
v & \rightarrow v^{\sigma}
\end{aligned}
$$

Hence, for every $x \in K$ and $v \in M_{K}$, we have $\left|x^{\sigma}\right|_{v^{\sigma}}=|x|_{v}$. Obviously $\sigma$ gives as well an isomorphism

$$
\sigma: K_{v} \rightarrow K_{v^{\sigma}}^{\sigma}
$$

Therefore $n_{v}=n_{v^{\sigma}}$, where $n_{v^{\sigma}}=\left[K_{v^{\sigma}}^{\sigma}: \mathbb{Q}_{v}\right]$. Then

$$
\begin{aligned}
\mathfrak{h}_{K^{\sigma}}\left(P^{\sigma}\right) & =\prod_{w \in M_{K^{\sigma}}} \max _{0 \leq i \leq n}\left\{\left|x_{i}^{\sigma}\right|_{w}^{n_{w} / q_{i}}\right\} \\
& =\prod_{v \in M_{K}} \max _{0 \leq i \leq n}\left\{\left|x_{i}^{\sigma}\right|_{v^{\sigma}}^{n_{v^{\sigma}} / q_{i}}\right\}=\prod_{v \in M_{K}} \max _{0 \leq i \leq n}\left\{\left|x_{i}\right|_{v}^{n_{v} / q_{i}}\right\}=\mathfrak{h}_{K}(\mathfrak{p})
\end{aligned}
$$

This completes the proof.

Given $\mathfrak{p} \in \mathbb{W} \mathbb{P}^{n}(K)$ as $\mathfrak{p}=\left[x_{0}, \ldots, x_{n}\right]$ the field of definition of $\mathfrak{p}$ is defined as

$$
\mathbb{Q}(\mathfrak{p}):=\mathbb{Q}\left(\left(\frac{x_{0}}{x_{i}}\right)^{\frac{q_{0}}{q}}, \ldots, 1, \ldots,\left(\frac{x_{n}}{x_{i}}\right)^{\frac{q_{n}}{q}}\right)
$$

Notice that $\mathbb{Q}(\mathfrak{p})$ is the field containing all the liftings of the field $\mathbb{Q}(\phi(\mathfrak{p}))$. In other words adjoining al the $q$-roots to the minimal field of definition $\mathbb{Q}(\phi(\mathfrak{p}))$ of $\phi(\mathfrak{p}) \in \mathbb{P}^{n}$.

Lemma 11. For any point $\mathfrak{p} \in \mathbb{W}_{\mathfrak{w}}^{n}(\overline{\mathbb{Q}})$, we have

$$
[\mathbb{Q}(\mathfrak{p}): \mathbb{Q}] \leq q \cdot[\mathbb{Q}(\phi(\mathfrak{p})): \mathbb{Q}]
$$

Proof. The proof follows from the fact that for every coordinate we have to possibly adjoin at most a $q$-th root of unity.

The following result is analogue to Northcott's theorem for weighted projective spaces. 
Theorem 1. Let $c_{0}$ and $d_{0}$ be constants and $\mathbb{W P}_{w}^{n}(\overline{\mathbb{Q}})$ the weighted projective space with weights $\mathfrak{w}=\left(q_{0}, \ldots, q_{n}\right)$. Then the set

$$
\left\{\mathfrak{p} \in \mathbb{W}_{w}^{n}(\overline{\mathbb{Q}}): \mathfrak{h}_{\mathbb{Q}}(\mathfrak{p}) \leq c_{0} \text { and }[\mathbb{Q}(\mathfrak{p}): \mathbb{Q}] \leq d_{0}\right\}
$$

contains only finitely many points.

Proof. The proof is a direct consequence of Northcott's theorem for projective spaces and Lem. 9, Let $q=q_{0} q_{1} \cdots q_{n}$ and consider the map $\phi: \mathbb{W}^{n}(k) \rightarrow \mathbb{P}^{n}(k)$ as defined in Eq. (19). From Northcott's theorem for projective spaces we have that if $C_{0}=c_{0}^{q}$ and $D_{0}=\frac{1}{q} d_{0}$ are constants and $\mathbb{P}^{n}(\overline{\mathbb{Q}})$ a projective space, then the set

$$
\left\{\phi(\mathfrak{p}) \in \mathbb{P}^{n}(\overline{\mathbb{Q}}): H(\phi(\mathfrak{p})) \leq C_{0} \text { and }[\mathbb{Q}(\phi(\mathfrak{p})): \mathbb{Q}] \leq D_{0}\right\},
$$

contains only finitely many points $\phi(\mathfrak{p})$. From Lem. 9 we have that

$$
\mathfrak{h}_{\mathbb{Q}}(\mathfrak{p})=H_{\mathbb{Q}}(\phi(\mathfrak{p}))^{\frac{1}{q}} \leq C_{0}^{\frac{1}{q}}=c_{0} .
$$

Also,

$$
[\mathbb{Q}(\mathfrak{p}): \mathbb{Q}] \leq q \cdot[\mathbb{Q}(\phi(\mathfrak{p})): \mathbb{Q}] \leq q \cdot D_{0}=d_{0} .
$$

Since $\phi$ is a finite degree map, then are only finitely many points $\mathfrak{p} \in \mathbb{W}_{\mathfrak{w}}^{n}(\overline{\mathbb{Q}})$ satisfying the above conditions. This completes the proof.

The following theorem is a more practical result especially from the computational point of view.

Corollary 4. There are finitely many absolutely normalized points $\mathfrak{p} \in \mathbb{W} \mathbb{P}_{\mathfrak{w}}^{n}(\overline{\mathbb{Q}})$ of bounded height. In other words,

$$
\left\{\mathfrak{p} \in \mathbb{W P}_{w}^{n}(\overline{\mathbb{Q}}): \mathfrak{h}_{\overline{\mathbb{Q}}}(\mathfrak{p}) \leq c_{0}\right\}
$$

is a finite set for any constant $c_{0}$.

Proof. Since $\mathfrak{p}$ is absolutely normalized then $\operatorname{wgcd}(\mathfrak{p})=1$. In this case $\mathbb{Q}(\mathfrak{p})=\mathbb{Q}$. The result follows from the above theorem.

Corollary 5. For any number field $K$, the set

$$
\left\{\mathfrak{p} \in \mathbb{W P}_{w}^{n}(K): \mathbb{Q}(\mathfrak{p}) \subset K \text { and } \mathfrak{h}_{K}(\mathfrak{p}) \leq c_{0}\right\},
$$

is a finite set.

Proof. Since $\mathbb{Q}(\mathfrak{p}) \subset K$ then $\mathfrak{p}$ is absolutely normalized $\mathbb{W}_{w}^{n}(K)$. The result follows from the above.

The next result is the analogue of what is called Kronecker's theorem for heights on projective spaces.

Lemma 12. Let $K$ be a number field, and let $\mathfrak{p}=\left[x_{0}: \cdots: x_{n}\right] \in \mathbb{W P}_{w}^{n}(K)$, where $\mathfrak{w}=\left(q_{0}, \ldots, q_{n}\right)$. Fix any $i$ with $x_{i} \neq 0$. Then $\mathfrak{h}(\mathfrak{p})=1$ if the ratio $x_{j} / \xi_{i}^{q_{j}}$, where $\xi_{i}$ is the $q_{i}$-th root of unity of $x_{i}$, is a root of unity or zero for every $0 \leq j \leq n$ and $j \neq i$. 
Proof. Let $\mathfrak{p}=\left[x_{0}: \cdots: x_{i}: \cdots: x_{n}\right] \in \mathbb{W}^{n}(K)$. Assume $x_{i} \neq 0$. Adjoin the $q_{i}$-th root of unity to $x_{i}$. Hence, let $x_{i}=\xi_{i}^{q_{i}}$ so that $w t\left(\xi_{i}\right)=1$. Without loss of generality we can divide the coordinates of $\mathfrak{p}$ by $\xi_{i}^{q_{j}}$, for $j \neq i$, and then we have

$$
\mathfrak{p}=\left[\frac{x_{0}}{\xi_{i}^{q_{0}}}, \ldots, 1, \ldots, \frac{x_{n}}{\xi_{i}^{q_{n}}}\right] .
$$

For simplicity let $\mathfrak{p}=\left[y_{0}: \cdots: 1: \cdots: y_{n}\right]$. If $y_{l}$ is a root of unity for every $0 \leq l \leq n$ and $l \neq i$ then $\left|y_{l}\right|_{v}=1$ for every $v \in M_{K}$. Hence, $\mathfrak{h}(\mathfrak{p})=1$.

\section{CONCLUding REMARKS}

The weighted greatest common divisors are a natural extension of the concept of greatest common divisors to weighted tuples. Wether the usual properties of the greatest common divisors for Dedekind Domains can be extended to the weighted greatest common divisors is a natural question that needs further study. Even more generally how the ideal calculus ([17, Appendix A] ) can be generalized in terms of weighted ideals? For example, can Lemma 5 and Lemma 6 in [17 be generalized for weighted greatest common divisors?

From the computational point of view it seems as there is no escape from the fact that to compute the weighted greatest common divisor one has to factor integers into primes. However, this is a problem that surely will be further investigated by computer algebra experts.

The theory of heights is fundamental in arithmetic geometry and heights for weighted projective spaces provide powerful tools to study rational points in such spaces or on weighted Abelian varieties. The weighted projective height has the basic properties of the projective height. Whether this can be used to fully develop an arithmetic geometry machinery over weighted projective spaces remains to be seen.

\section{REFERENCES}

[1] Mauro Beltrametti and Lorenzo Robbiano, Introduction to the theory of weighted projective spaces, Exposition. Math. 4 (1986), no. 2, 111-162. MR.879909

[2] L. Beshaj and M. Polak, On hyperelliptic curves of genus 3, Algebraic curves and their applications, 2019, pp. 161-173. MR3916739

[3] Lubjana Beshaj and Scott Guest, The weighted moduli space of binary sextics, Algebraic curves and their applications, 2019, pp. 33-44. MR3916733

[4] Gilberto Bini, Quotients of hypersurfaces in weighted projective space, Adv. Geom. 11 (2011), no. 4, 653-667. MR2852925

[5] Enrico Bombieri and Walter Gubler, Heights in Diophantine geometry, New Mathematical Monographs, vol. 4, Cambridge University Press, Cambridge, 2006. MR2216774

[6] Alexandru Buium, Weighted projective spaces as ample divisors, Rev. Roumaine Math. Pures Appl. 26 (1981), no. 6, 833-842. MR627828

[7] A. Clingher, A. Malmendier, and T. Shaska, Configurations of 6 lines and string dualities, Communications in Mathematical Physics to appear (2019).

[8] An-Wen Deng, Rational points on weighted projective spaces, arXiv preprint math (1998).

[9] Igor Dolgachev, Weighted projective varieties, Group actions and vector fields (Vancouver, B.C., 1981), 1982, pp. 34-71. MR704986

[10] Gerhard Frey and Tony Shaska, Curves, Jacobians, and cryptography, Algebraic curves and their applications, 2019, pp. 279-344. MR3916746

[11] Marc Hindry and Joseph H. Silverman, Diophantine geometry, Graduate Texts in Mathematics, vol. 201, Springer-Verlag, New York, 2000. An introduction. MR.1745599

[12] Jun-ichi Igusa, Arithmetic variety of moduli for genus two, Ann. of Math. (2) 72 (1960), 612-649. MR0114819 
[13] Irving Kaplansky, Commutative rings, The University of Chicago Press, 1974.

[14] A. Malmendier and T. Shaska, The Satake sextic in F-theory, J. Geom. Phys. 120 (2017), 290-305. MR 3712162

[15] Andreas Malmendier and Tony Shaska, A universal genus-two curve from Siegel modular forms, SIGMA Symmetry Integrability Geom. Methods Appl. 13 (2017), Paper No. 089, 17. MR.3731039

[16] Jorgo Mandili and Tony Shaska, Computing heights on weighted projective spaces, Algebraic curves and their applications, 2019, pp. 149-160. MR.3916738

[17] Aharon Razon, Primitive recursive decidability for large rings of algebraic integers., Albanian J. Math. 13 (2019), no. 1, 1-91 (English).

[18] Carlo A. Rossi, Weighted projective spaces and minimal nilpotent orbits, Represent. Theory 12 (2008), 208-224. MR2403559

[19] T. Shaska and L. Beshaj, Heights on algebraic curves, Advances on superelliptic curves and their applications, 2015, pp. 137-175. MR.3525576

[20] Joseph H. Silverman, Generalized greatest common divisors, divisibility sequences, and Vojta's conjecture for blowups, Monatsh. Math. 145 (2005), no. 4, 333-350. MR2162351 